\title{
The effects of urea, guanidinium chloride and sorbitol on porphyrin aggregation: Molecular dynamics simulation
}

\author{
MARYAM GHADAMGAHI ${ }^{\mathrm{a}}$ and DAVOOD AJLOO ${ }^{\mathrm{a}, \mathrm{b} *}$ \\ ${ }^{a}$ School of Chemistry, ${ }^{b}$ Institute of Biological Science, Damghan University, Damghan, Iran \\ e-mail: M_ghadamgahi@st.du.ac.ir; ajloo@du.ac.ir
}

MS received 19 July 2012; revised 23 November 2012; accepted 21 January 2013

\begin{abstract}
This paper compares the inhibition effect of porphyrin aggregation in the presence of urea, guanidinium chloride (Gdn) and sorbitol by molecular dynamics simulation. It demonstrates that porphyrin aggregation increases in sorbitol, but decreases towards addition of urea and Gdn. It shows that urea, Gdn and sorbitol can have a large effect - positive or negative, depending on the concentration - on the aggregation of the porphyrin. The effect of urea, Gdn and sorbitol on porphyrin aggregation has been inferred from the effect of these solutes on the hydration layer of porphyrin. It appears that the Gdn is more suitable than urea for decreasing the hydration layer of porphyrin while several osmolites like sorbitol are known to increase hydration layer and thus might stabilize the porphyrin aggregation. Results of radial distribution function (RDF), distributed atoms or molecules around target species, indicated that the increase and exclusion of solvent around porphyrin by osmolytes and Gdn would affect significantly on porphyrin aggregation. There was a sizeable difference in potency between the Gdn and urea, with the urea being less potent to decrease hydration layer and porphyrin aggregation.
\end{abstract}

Keywords. Porphyrin; molecular dynamics simulation; urea; sorbitol; radial distribution function; aggregation.

\section{Introduction}

The importance of porphyrins as therapeutic drugs and targeting agents has been widely recognized. ${ }^{1}$ Porphyrins have been widely studied as sensitizers of living organisms, including humans, in light-induced reactions. ${ }^{2}$ Porphyrin-photosensitized processes can be very harmful and cause irreversible damage to cells, such as human porphyria or skin photo damage (erythema and cancer). ${ }^{3}$ On the other hand, the use of sensitizers is currently finding biomedical application in the treatment of several diseases, including photodynamic therapy (PDT) of malignant tumours, ${ }^{4}$ photosensitized medal plaque destruction ${ }^{5}$ and curing of psoriatic lesions. ${ }^{6}$

The major driving forces operating in porphyrin aggregation are considered to be $\pi-\pi$ stacking, ${ }^{7}$ intermolecular hydrogen bonding interactions, ${ }^{8}$ and van Der Waals interactions. ${ }^{9}$ These interactions have been effectively modulated by structural changes of the involved species, such as the change of groups around porphyrin macro cycles, or the nature and the morphology of derivatives. Aggregation behaviour of porphyrin can

*For correspondence depend on the size, shape and hydrophobicity of substitutes. The physico-chemical and photophysical properties of porphyrins are associated to their structural characteristics that allow in many cases an effective interaction with the membrane matrix, involving localization of these molecules in different membrane environments. The physico-chemical properties of porphyrins can be deeply influenced by the interaction with micelle and other molecules such as urea and Gdn. ${ }^{10}$ The factors influencing the affinity of porphyrins for biological membranes, including the dye hydrophobicity, ${ }^{11}$ the charge and aggregation state, ${ }^{12}$ the $\mathrm{pH}$ of the medium and the physicochemical properties of the dye environment. ${ }^{13}$ These factors determine the differences in the photophysical properties of porphyrins in biological membranes. Particular attention is paid to the porphyrin aggregation state and distributed solvent around porphyrin.

Owing to the above-outlined considerations, this investigation will be concerned mainly with the molecular dynamics simulation study of sorbitol, urea and Gdn effect on porphyrin aggregation. Studies of parameters such as water distribution as well as concentration effect of porphyrin will be particularly emphasized. Owing to the extensive literature existing in this 
field, only porphyrins of phototherapeutic interest will be considered. Urea and Gdn have essential but different effects on the aggregation of porphyrin, which can be utilized to control the aggregation behaviours of porphyrin, such as aggregate size, and furthermore, the geometric arrangement of porphyrin monomer in aggregate. Porphyrin forms higher aggregates in the presence of sorbitol and form lower aggregate in the presence of urea and Gdn. The use of realistic co-solvents in MD simulations of proteins is uncommon. There have been few such studies reported to date: ubiquitin in $60 \%$ methanol ${ }^{14}$ and barnase in $8 \mathrm{M}$ urea. ${ }^{15}$ Thus, there is still a need for further investigation of urea's effect on other compounds such as porphyrin aggregation, and it is now possible to perform much longer simulations to view the entire reaction coordinate. In preparation for the simulations described here, we have addressed the effect of urea on water structure and dynamics, particularly around porphyrin. Urea has effect on water structure with respect to radial distribution functions results. Consequently, we used the radial distribution function as a metric to distinguish the solutions and the aggregation, because they can reveal subtle differences not seen in other ensemble-averaged properties. In the hydration shell of pophyrin molecules, these waters are restricted because of their efforts to maximize interactions with neighbouring waters while minimizing interactions with porphyrin. ${ }^{16}$

Thus, urea appears to better solubilize hydrophobic solutes by, in effect, perturbing water and thereby preloading the solvent to accept non-polar groups by subtle disruption of water's preferred structure and reorientation of the waters around its polar atoms. At higher concentrations, there are more direct interactions between the urea and solute. Therefore, our model studies suggest that urea and Gdn act directly and sorbitol acts indirectly on model compound. We used the MD simulation to study decrease of aggregation in the presence of Gdn and urea. Urea and Gdn, which increase the surface free energy of water, are preferentially bound to porphyrin at high concentrations. Conversely, osmolites like sorbitol, which decrease the surface free energy of water, induce porphyrin preferential hydration and increase porphyrin hydration layer and far from porphyrin aggregate in high concentrations. ${ }^{17,18}$

Based on these experiments, two different mechanisms have been proposed: an 'indirect mechanism' in which urea is presumed to disrupt the structure of water, thus making hydrophobic groups more readily solvated, ${ }^{19}$ and a 'direct mechanism' in which urea interacts either directly with the backbone of model molecule, ${ }^{20}$ via hydrogen bonds and other electrostatic interactions, ${ }^{21}$ or directly with the porphyrin through more favourable van der Waals attractions as compared with water, ${ }^{22,23}$ or both. The 'direct electrostatic mechanism' suggests that urea and Gdn interact directly with the protein backbone, via hydrogen bonds, and other electrostatic interactions with charged and polar side chains predominantly. Direct binding of urea to the porphyrin is best explained by the RDF curves of this study. However, the 'direct van der Waals mechanism' suggests that urea destabilizes porphyrin aggregation predominantly through its stronger dispersion interactions with the water than porphyrin. In this article, we use the MD trajectory of porphyrin in various concentrations of urea and guanidium generated on the massively parallel computer, to analyse the destabilization by urea, which has been observed experimentally. We find that destabilization of porphyrin aggregation occurs predominantly by the direct interactions of urea and Gdn with the porphyrin via dispersion or van der Waals interactions and increasing porphyrin aggregation occur by indirect interaction of sorbitol with porphyrin. The more favourable van der Waals interactions of urea relative to water with porphyrin attract urea to the porphyrin surface. We also find that urea disrupts the water structure or its hydrogen-bonded network because of its interaction with water, and it also changes much of the orientational dynamics of water. The preferential hydration of porphyrin was considered in the presence of Gdn, urea and sorbitol. The high values of preferential hydration found in sorbitol, suggest that they should stabilize porphyrin aggregated structure. ${ }^{24}$

\section{Materials and methods}

\subsection{Molecular dynamics simulations}

The structures of porphyrin, urea, Gdn and sorbitol were drawn using Hyperchem 7 software. Structures of studied molecules are presented in scheme 1 . These structures were pre-optimized with Molecular Mechanics Force Field $(\mathrm{MM}+)$ and final geometries were obtained with the semi-empirical AM1 method in the Hyperchem program. All calculations were carried out at the restricted Hartree-Fock level. The molecular structures were optimized using the Polak-Ribiere algorithm until the root mean square gradient was $0.001 \mathrm{kcal} \mathrm{mol}^{-1}$. Force field and geometries parameters were generated using PRODRG2 server (http:// davapc1.bioch.dundee.ac.uk/cgi-bin/prodrg_beta). A cubic simulation box of the volume $4.46 \times 4.36 \times$ $4.32 \mathrm{~nm}^{3}$ was made and six porphyrins were placed randomly in this box. 20, 40, 60 and 80 urea, Gdn and sorbitol molecules were added. Then, water molecules were randomly added into the simulation box and 


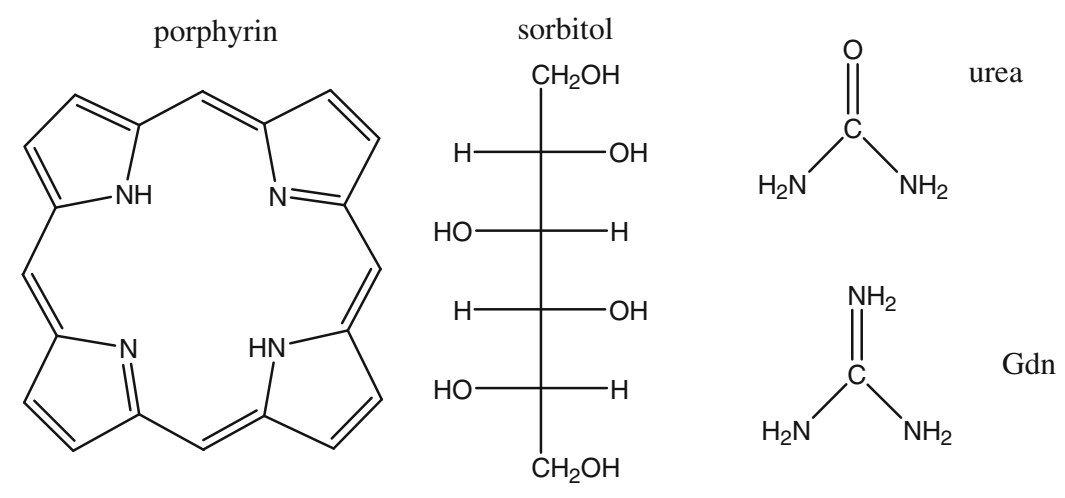

Scheme 1. Structure of porphyrin, sorbitol, urea and guanidinium chloride.

initial configurations were minimized using steepest descent algorithm with 5000 integration step. All MD simulations were carried out using the GROMACS 4.5.0. Structure parameters include RDF, order parameter, distance, radius of gyration, rate constant and RMSD were obtained and averaged. One of the trajectories in the presence of $20 \mathrm{Gdn}$, urea and sorbitol was performed for $40 \mathrm{~ns}$ to confirm results of $20 \mathrm{~ns}$ simulation time. Averaged data's of distance between porphyrin were transferred in to Origin (version 5) software and were fitted by first order exponential decay to calculate the decay time of aggregation and rate constant was obtained. All MD simulations were carried out using the GROMOS 96-43al force field, and simulation temperature of $300 \mathrm{~K}$. The non-bonded interaction was switched to zero at a cut-off of $9 \AA$. The simple point charge (SPC) model was used to describe water. A different time step was used to integrate the equations of motion with the Verlet algorithm. The longrange electrostatic interactions were treated with the particle mesh Ewald method. Temperatures and pressures were controlled by a Nose-Hoover thermostat $[49,50]$ and Parrinello-Rahman barostate [51] with coupling constants of 0.1 and 0.5 , respectively.

The calculations were performed using 5 quad core parallel computers including 40 processor units. The computer applied the Rocks cluster networking and Centos operating systems. Results were calculated by three repeated and averaged trajectory of simulation.

\subsection{Data analyses}

The conformational changes of the porphyrin during MD simulations were monitored by the root-meansquare derivations (RMSD). The RMSD value, a measure of molecular mobility, is calculated by translating and rotating the coordinates of the instantaneous structure to superimpose the reference structure with a maximum overlap. The RMSD is defined as

$$
R M S D=\sqrt{\frac{\sum_{i=1}^{N} m_{i}\left(r_{i}-r_{i}^{0}\right)}{\sum_{i=1}^{N} m_{i}}}
$$

where $m_{i}$ is the mass of atom $i . r_{i}$ and $r_{i}^{\circ}$ are the coordinates of atom $i$ during MD simulations and at its reference state, respectively. RMSDs were calculated, for the trajectories, from the starting structures of porphyrin as a function of time. In the all systems, RMSDs reach a stable value within the first nano second of all the analyses.

The radial distribution function $g(r)$ is the density of probability for finding a particle at distance $r$ from the reference particle. RDF between particles of type A and $\mathrm{B}$ is defined in the following terms

$$
\begin{aligned}
R D F & =\frac{\langle\rho B(r)\rangle}{\langle\rho B\rangle_{\text {local }}} \\
& =\frac{1}{\langle\rho B\rangle_{\text {local }}} \frac{1}{N_{A}} \sum_{i \in A}^{N_{A}} \sum_{j \in B}^{N_{B}} \frac{\delta\left(r_{i j}-r\right)}{4 \pi r^{2}}
\end{aligned}
$$

where $<B(r)>$ is the particle density of type $\mathrm{B}$ at a distance $r$ around particles $\mathrm{A}$, and $\langle B\rangle_{\text {local }}$ is the particle density of type B averaged over all spheres around particles A with radius $r_{\max }$. Usually the value of $r_{\max }$ is half of the box length.

g _gyrate compute a rough measure for the compactness of a structure. The radius of gyration $\left(R_{\mathrm{gyr}}\right)$ for porphyrins defined as the mass-weighted geometric mean of the distance of each atom from the porphyrin's 
centre of mass. The radius of gyration for the porphyrins was computed using the standard formula.

$$
R_{g y r}=\sqrt{\frac{\sum m_{i} r_{i}^{2}}{\sum m_{i}}},
$$

where $r_{\mathrm{i}}$ is the distance of atom $i$ from the centre of mass of the porphyrin, and $m_{\mathrm{i}}$ is its mass.

The distance between mass centres of porphyrins have been also calculated. Averaged data of distance between porphyrins transferred in to Origin (version 5) software. Rate constants determined by computer fitting the kinetic data to the first order exponential decay and rate constant obtained from the inverse relation of rate with relaxation time as following.

$$
D=D_{o} e^{-k t}=D_{o} e^{-t / \tau},
$$

where $D$ and $D_{o}$, are distance between porphyrin at $t=t$ and $t=0$, while $k$ and $\tau$ are rate constant and relaxation time, respectively.
The order parameter is obtained by the following equation

$$
s_{z}=\frac{3}{2}\left\langle\cos ^{2} \theta_{z}\right\rangle-\frac{1}{2},
$$

where $\theta_{z}$ is the angle between the z-axis of the simulation box and the molecular axis under consideration. The brackets imply averaging over time and molecules, so it can compute the order parameter per atom. ${ }^{25}$

\section{Results and discussion}

\subsection{Porphyrin aggregation}

The stacking interactions of porphyrin offer a versatile application in future and allow preparation of porphyrin for use in future. This investigations point out that porphyrin aggregates in aqueous solution. To this end, porphyrin aggregation in aqueous solutions was investigated as a function of the concentration by molecular dynamics simulation. $6(0.02 \mathrm{M})$ and $12(0.08 \mathrm{M})$
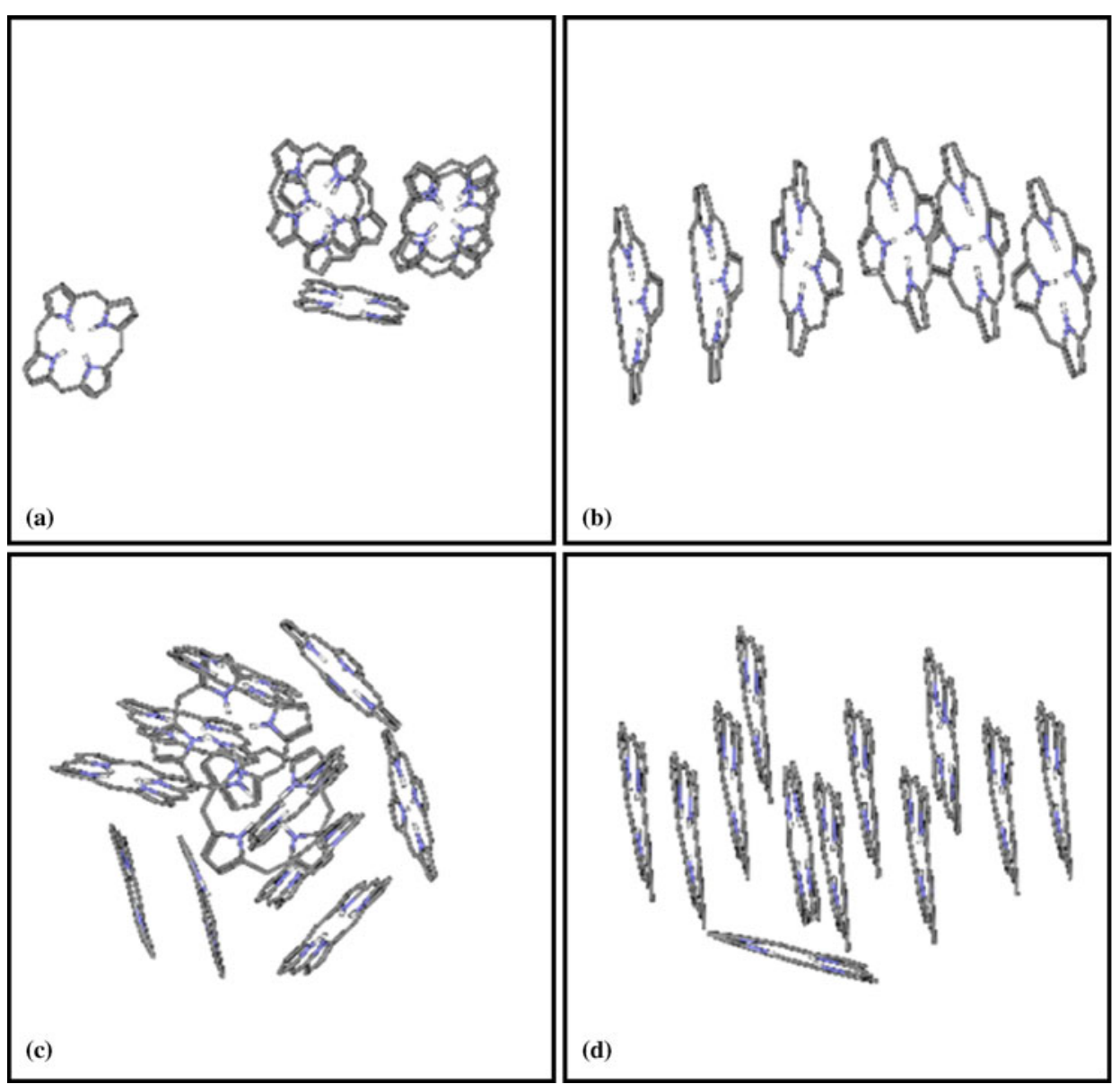

Figure 1. Snapshot of molecular picture taken by DS Visualizer program for two concentrations of $0.02(\mathbf{a}, \mathbf{b})$ and $0.08 \mathrm{M}(\mathbf{c}, \mathbf{d})$ without $(\mathbf{a}, \mathbf{c})$ and with $(\mathbf{b}, \mathbf{d})$ simulated annealing, respectively after $20 \mathrm{~ns}$. 
porphyrin was added to simulation box and increase of aggregation by concentration was observed.

In order to have a better aggregation pattern, simulated annealing was done. Simulated annealing is a special case of MD or MC simulation, in which the temperature is gradually increased or reduced during the simulation. Often, the system is first heated and then cooled. Thus, the system is given the opportunity to surmount energetic barriers in a search for conformations with energies lower than the local-minimum energy found by energy minimization. One of the applications of MD is involved in utilization of MD, often with simulated annealing protocols, to determine or refine structures with data obtained from experiments. ${ }^{26}$ In one investigation 6 and 12 porphyrin were simulated at $300 \mathrm{~K}$ and in the other simulated annealing was used and for this case temperature increased from 250 to $450 \mathrm{~K}$ during $20 \mathrm{~ns}$. It means that in each 500 step temperature increased $5 \mathrm{~K}$ by simulated annealing to have a better aggregation pattern and for comparison purpose.

Snapshot of molecular picture was taken by DS Visualizer program after simulation for two concentrations of 0.02 and $0.08 \mathrm{M}$ that are presented in figure 1a and c. Water molecules were removed for clarity. Figure 1a-d show the picture of $0.02(a, b)$ and 0.08 (c, d) M porphyrin without $(a, c)$ and with $(b, d)$ simulated annealing, respectively. Results show that the simulated annealing causes more order between aggregated porphyrins.

This figure shows that there are many more ordered porphyrin molecules aggregated near each other in the simulated annealing case. We interpret these effects as being due to the formation of rather fragile aggregation networks i.e., higher order assemblies involving interactions of aggregates. We were able to increase these higher order structures in the simulation runs by simulated annealing.

Order parameter versus time was also obtained for two concentrations of porphyrin. Diagram of $S_{j}$ versus time of simulation were obtained to compare order parameter which is depicted in figure 2a. It was observed from pictures that in systems with simulated annealing, order parameter of aggregated forms increase.

The comparison of order parameter for two concentrations was obtained and picture is not shown here. It
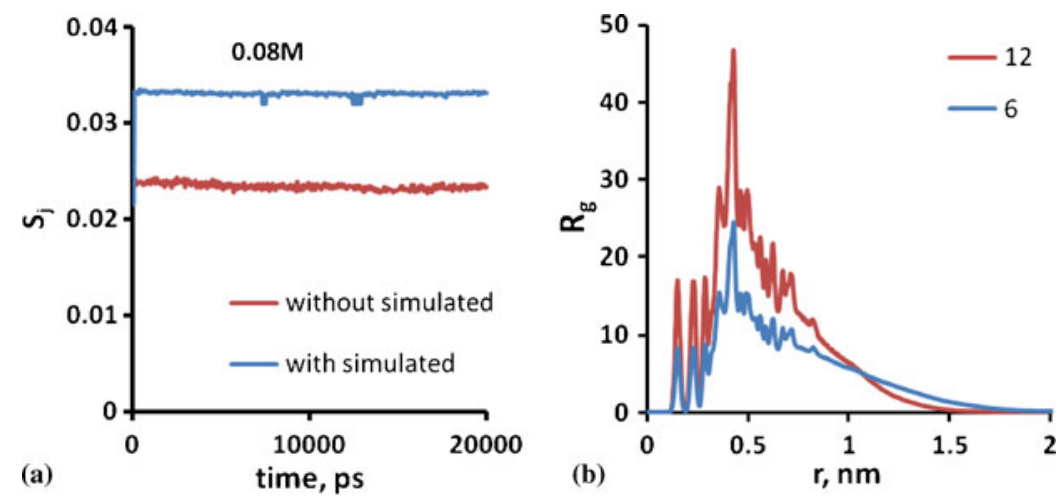

Figure 2. (a) Order parameter $\left(S_{j}\right)$ versus time for concentrations of $0.08 \mathrm{M}$ porphyrin with and without simulated annealing. (b) RDF diagrams of porphyrins versus distance for two concentrations of 0.02 and $0.08 \mathrm{M}$ porphyrin.
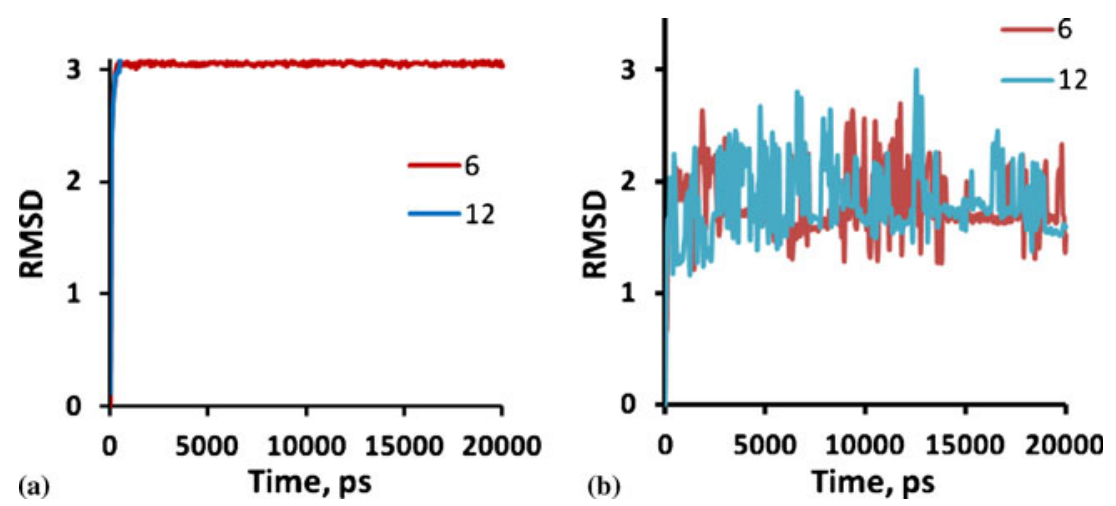

Figure 3. RMSD of 0.02 (6) and 0.08 (12) M porphyrin (a) with and (b) without simulated annealing. 
was also oserved that by increasing porphyrin concentration, order parameter can be increased.

The radial distribution function of porphyrin around each other versus distance is shown in figure $2 b$ for two concentrations of porphyrin. It can be seen that $\mathrm{RDF}$ is increased by increasing the concentration. An increase of RDF in aqueous solution with an increase in porphyrin content is usually viewed due to porphyrin aggregation. It is expected from the above results that porphyrin forms more ordered aggregates in higher concentration. The results of MD simulation of porphyrin represent more tendencies of concentrate porphyrin for aggregation in comparison with others.

Figure 3 shows the RMSD of 0.02 (6) and $0.08 \mathrm{M}$ (12) porphyrin in the $20 \mathrm{~ns}$ time interval with and without simulated annealing. It shows that the system reaches a stable state after about 500 ps. It also shows that more concentrate system has more structural changes.

\subsection{Concentration effect}

Interactions of Gdn, urea and sorbitol with porphyrin have been analysed using molecular dynamics simulation. Study of the porphyrin aggregation was carried out in the presence of different concentration of Gdn, urea and sorbitol. All MD simulations and calculations were done during $20 \mathrm{~ns}$ and one of the trajectories was simulated during $40 \mathrm{~ns}$. The structure information such as RMSD and RDF were obtained at $300 \mathrm{~K}$. Root mean square deviations (RMSD) of the systems in the presence of 20 to $80 \mathrm{Gdn}$, urea and sorbitol was obtained. Figure $4 \mathrm{a}-\mathrm{c}$ show the RMSD of system in the presence of 20 to $80 \mathrm{Gdn}$, urea and sorbitol molecules. It shows that the system reaches a stable state after about 500 ps. The figures also show that porphyrin has more structural changes (RMSD) in the presence of higher concentration than low concentration. When concentration is increased, the RMSD and structural changes also increased. Figure 4d compares RMSD of system in the presence of $20 \mathrm{Gdn}$, urea and sorbitol which has been selected randomly. It shows more structural changes of system in the presence of Gdn than urea. It has been shown that the system with Gdn and sorbitol has more structural changes than the urea. The compared curves show strong effect for Gdn than urea to affect system and porphyrin aggregation.

Radius of gyration for porphyrins in $20 \mathrm{~ns}$ time interval in the presence of 20 to $80 \mathrm{Gdn}$, urea and
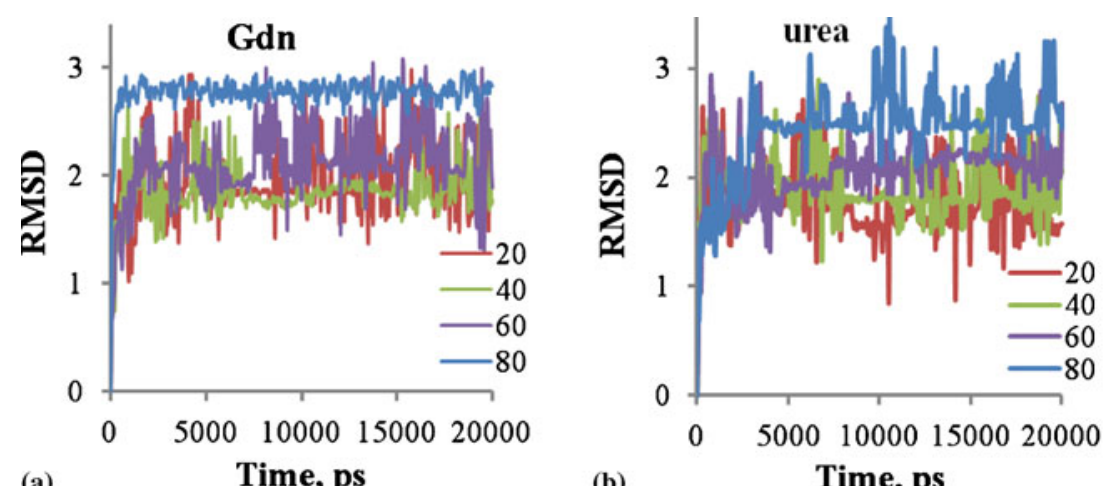

(a)

Time, ps
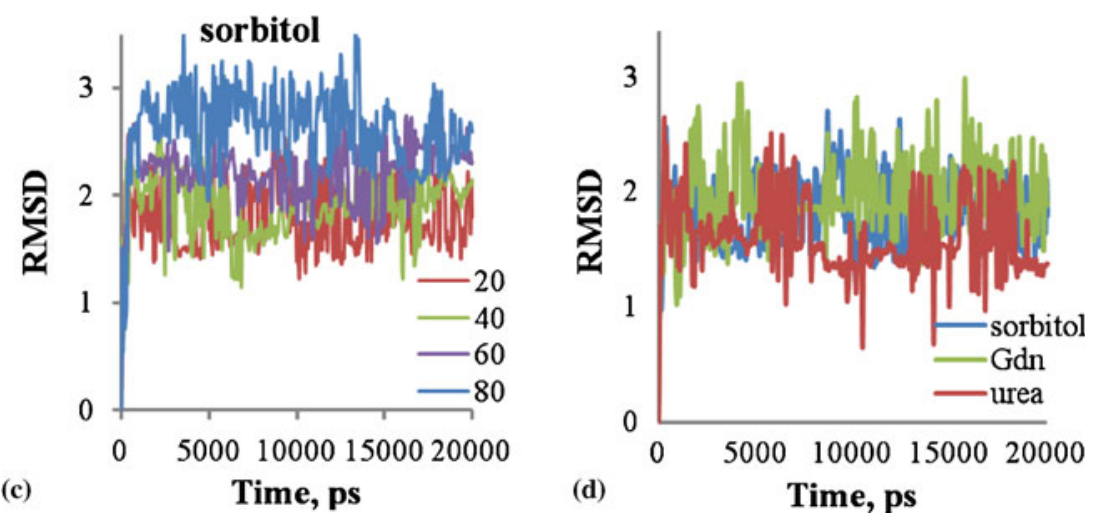

Figure 4. RMSD diagrams of porphyrin in the presence 20 to 80 . (a) Gdn, (b) urea and (c) sorbitol. (d) Comparing the RMSD diagrams of system in the presence of $20 \mathrm{Gdn}$, urea and sorbitol. 
sorbitol molecules were obtained and compared. Figure 5a shows the radius gyration of porphyrins in $20 \mathrm{~ns}$ time interval in the presence of 20 to $80 \mathrm{Gdn}$. It shows increase of radius gyration of porphyrin in the presence of high concentration of Gdn. This proves that the porphyrin aggregation has been reduced and it is obvious that radius gyration of porphyrin in system with 80 molecules is increased more due to more decrease of porphyrin aggregation. Radius gyration of porphyrin in the presence of high concentration of sorbitol decrease and the picture is not shown here. This proves that the porphyrin aggregation has been increased by sorbitol and sorbitol has stabilized porphyrin aggregation. Figure $5 \mathrm{~b}$ compares radius gyration of porphyrin in the presence of $20 \mathrm{Gdn}$, urea and sorbitol molecules. Radius gyration of porphyrin is more in the presence
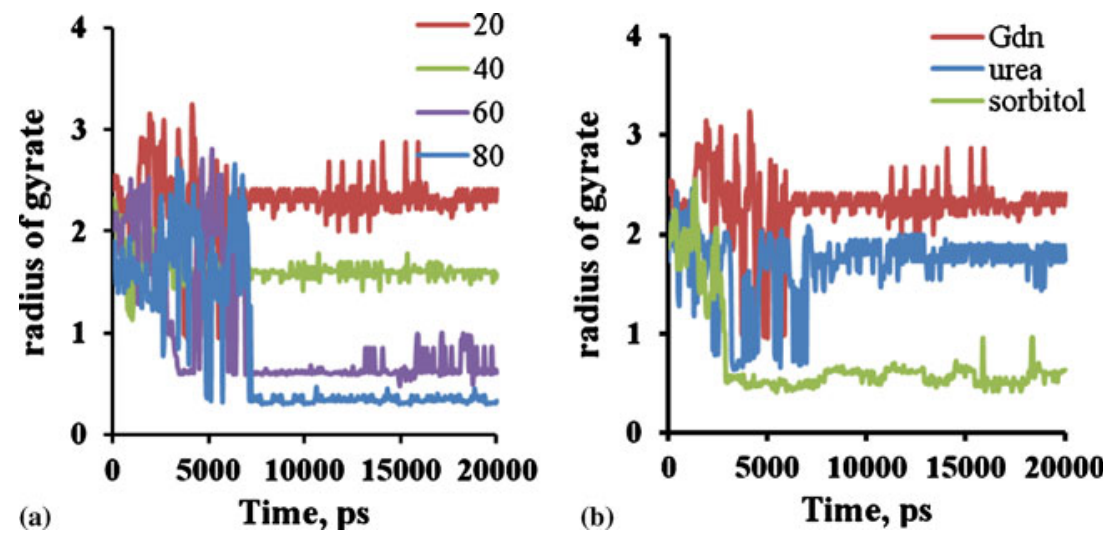

Figure 5. Radius of gyration for porphyrins in the last $20 \mathrm{~ns}$ time interval in the presence of 20 to 80 molecules of (a) Gdn. (b) Comparison of radius of gyration for porphyrins in the presence $20 \mathrm{Gdn}$, urea and sorbitol.
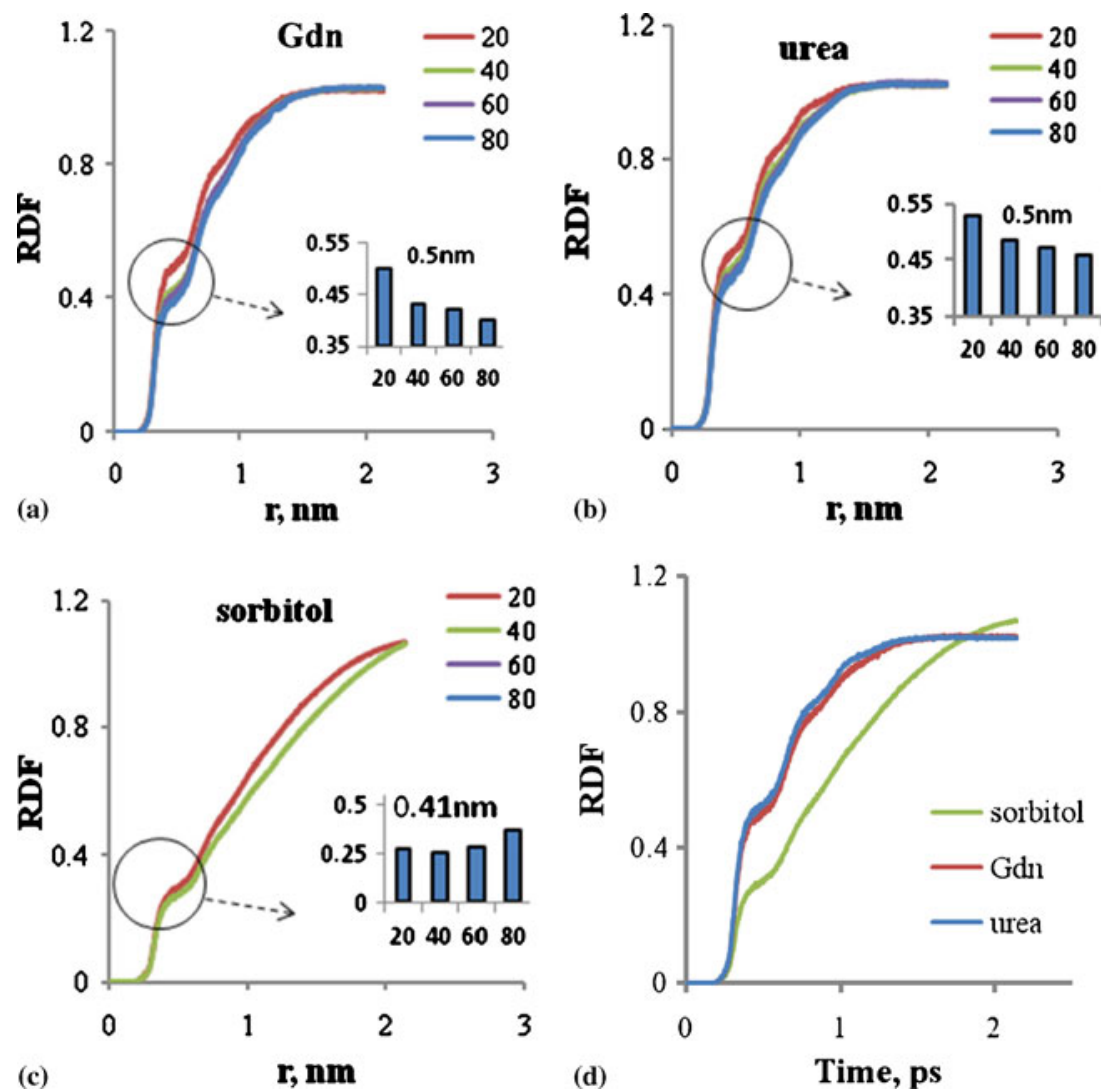

Figure 6. RDF of porphyrin-solvent in the $20 \mathrm{~ns}$ time interval in the presence of 20 to 80. (a) Gdn, (b) urea and (c) sorbitol. (d) Comparing the RDF of porphyrin-solvent in the $20 \mathrm{~ns}$ time interval in the presence of $20 \mathrm{Gdn}$ and urea. 
of Gdn, urea and sorbitol respectively. This proves that the porphyrin aggregation has been increased more in the presence of sorbitol and decreased more in the presence of Gdn than urea. It shows that aggregation of porphyrin is more in the presence of sorbitol, urea and Gdn respectively.

Radial distribution function (RDF) is a criterion for the distribution of atoms, molecules or other species around target species. The radial distribution function of solvent around porphyrin vs. distance is shown in figure $6 \mathrm{a}-\mathrm{c}$ in various concentrations of Gdn, urea and sorbitol, respectively. From these, one can infer that in general, as the concentration of Gdn and urea increase, the RDF of solvent around porphyrin decrease and with the increase of sorbitol concentration (figure $6 \mathrm{c}$ ), the RDF of solvent around porphyrin increase.

In high concentration, both Gdn and urea cause solvent exclusion from porphyrin and this effect is more for Gdn than urea but RDF is increased for sorbitol and by increasing the sorbitol concentration the RDF is increased. Existence probability of solvent around porphyrin in the presence of 20 to 80 sorbitol is more than 20 to $80 \mathrm{Gdn}$. The increase of RDF in aqueous solution with a decrease in sorbitol content is usually observed.
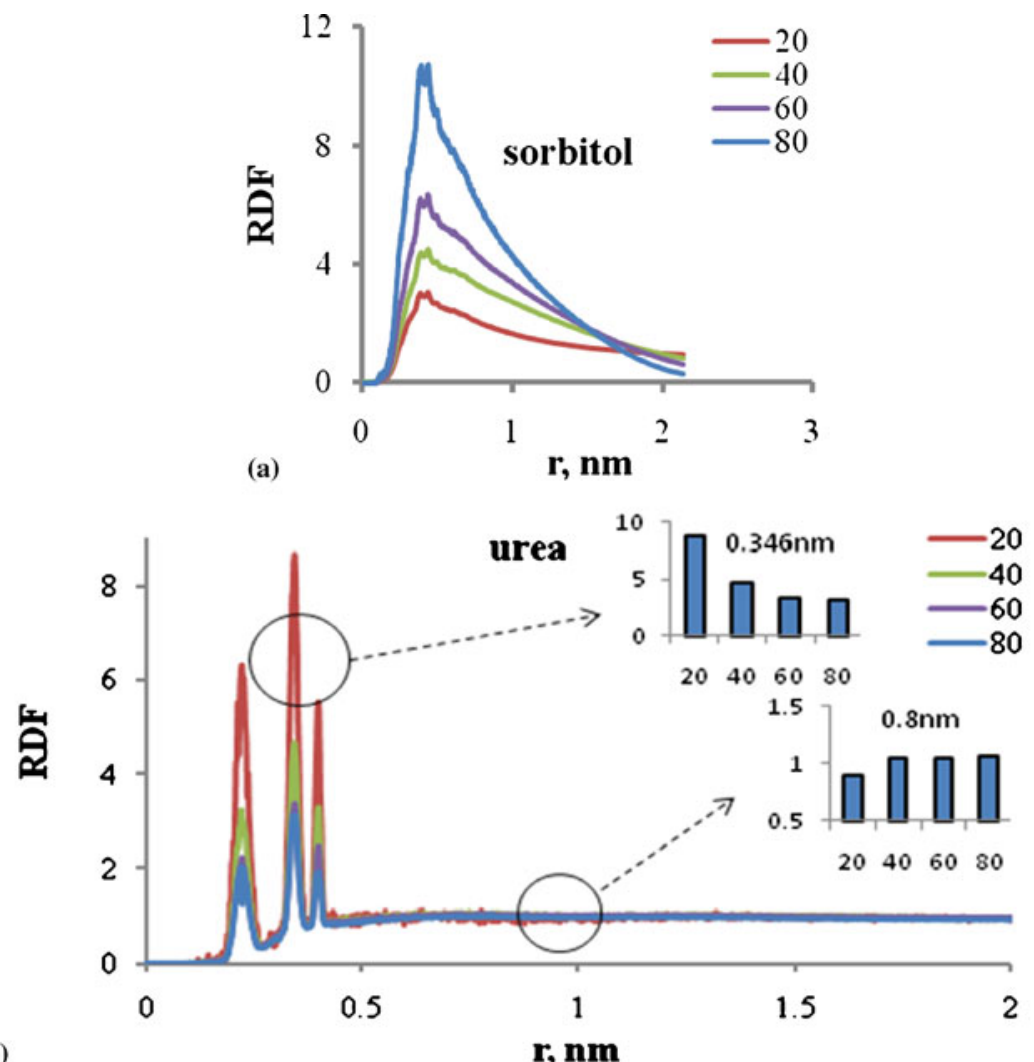

(b)

$\mathbf{r}, \mathbf{n m}$

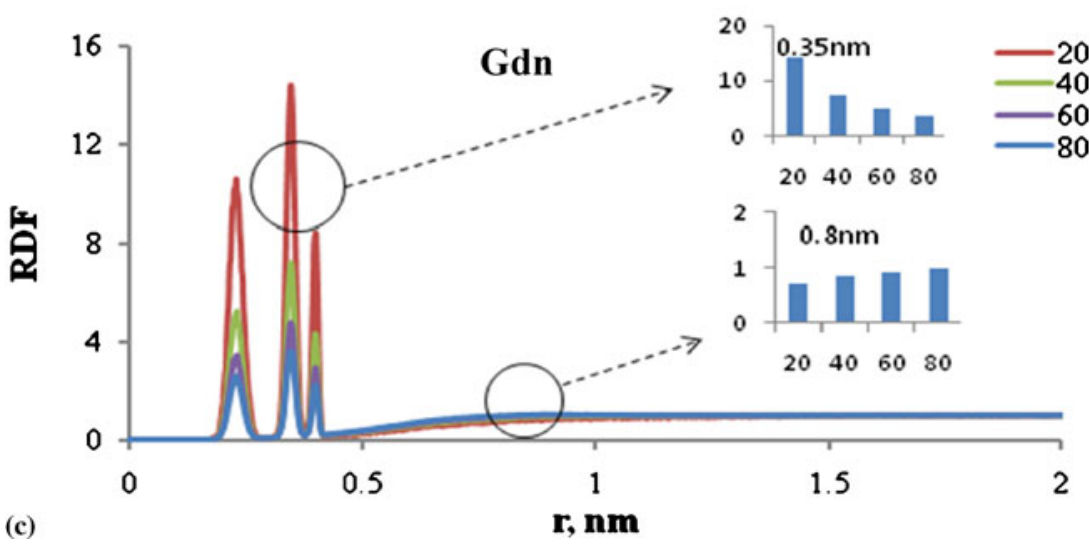

Figure 7. Calculated RDF of (a) sorbitol-sorbitol, (b) urea-urea and (c) Gdn-Gdn by molecular dynamics in the presence of 20 to 80 sorbitol, urea and Gdn molecules. 
The results show that solvent molecules are increased in hyrdation layer around porphyrin and this is due to sorbitol molecules that become excluded from porphyrinsolvent surface. RDF values of porphyrin-solvent has been obtained, drawn and included in figures in $0.5 \mathrm{~nm}$ distance. These figures show more effect of Gdn for reduction of hydration layer around porphyrin. We can conclude from RDF diagrams that a thin hydration layer is formed on porphyrin surface in high concentration of sorbitol and this layer is reduced more in the presence of Gdn than urea.

Figure $6 \mathrm{~d}$ compares the RDF of porphyrin-solvent in the $20 \mathrm{~ns}$ time interval in the presence of $20 \mathrm{Gdn}$ and urea. Probability of occurrence of solvent around porphyrin is compared and this probability is more for sorbitol, urea and Gdn. As a consequence Gdn causes more solvent exclusion from porphyrin. It can be seen that RDF is decreased for Gdn more than urea by increasing the Gdn and urea concentration. Moreover, the results further suggest that RDF parameters of porphyrin-solvent provide a reasonable description for more decrease of hydration layer by Gdn than urea.

Calculated RDF of sorbitol around sorbitol, ureaurea and Gdn-Gdn by molecular dynamics in the presence of 20 to 80 sorbitol, urea and Gdn molecules are depicted in figure $7 \mathrm{a}-\mathrm{c}$, respectively. The results show an increase in RDF while increasing the sorbitol concentration, thus show self-aggregation of sorbitol in high concentrations. These calculations results revealed different tendencies of aggregate formations for three different molecules. The presence of sorbitol causes the stabilization of porphyrin aggregation, increase of hydration layer around porphyrin and results in a self aggregation with an increase in the sorbitol concentration which is obvious in figure $7 \mathrm{a}$. Three observed peak in figure $7 \mathrm{~b}$ and $\mathrm{c}$ is directly related to the $\mathrm{N}-$ $\mathrm{N}$ and $\mathrm{N}-\mathrm{H}$ intermolecular interaction. ${ }^{24}$ In $0.346 \mathrm{~nm}$ distance, RDF is decreased but in long distances of $0.8 \mathrm{~nm}$ RDF is increased. It means that at long distances urea behaves similar to osmolites and tend to aggregate in high concentrations. In $0.35 \mathrm{~nm}, \mathrm{RDF}$ is decreased for Gdn and in $0.8 \mathrm{~nm}$ RDF is increased and Gdn also tend to aggregate. We can conclude from all the above diagrams that probability of sorbitol presence around themselves increases in higher concentration and sorbitol increases the hydration layer around porphyrin and therefore increases porphyrin aggregation while Gdn and urea decrease this hydration layer around porphyrin and they move toward porphyrin and reduce porphyrin aggregation and this effect is more for Gdn than urea. Probability of sorbitol presence around porphyrin decreases by concentration and probability of
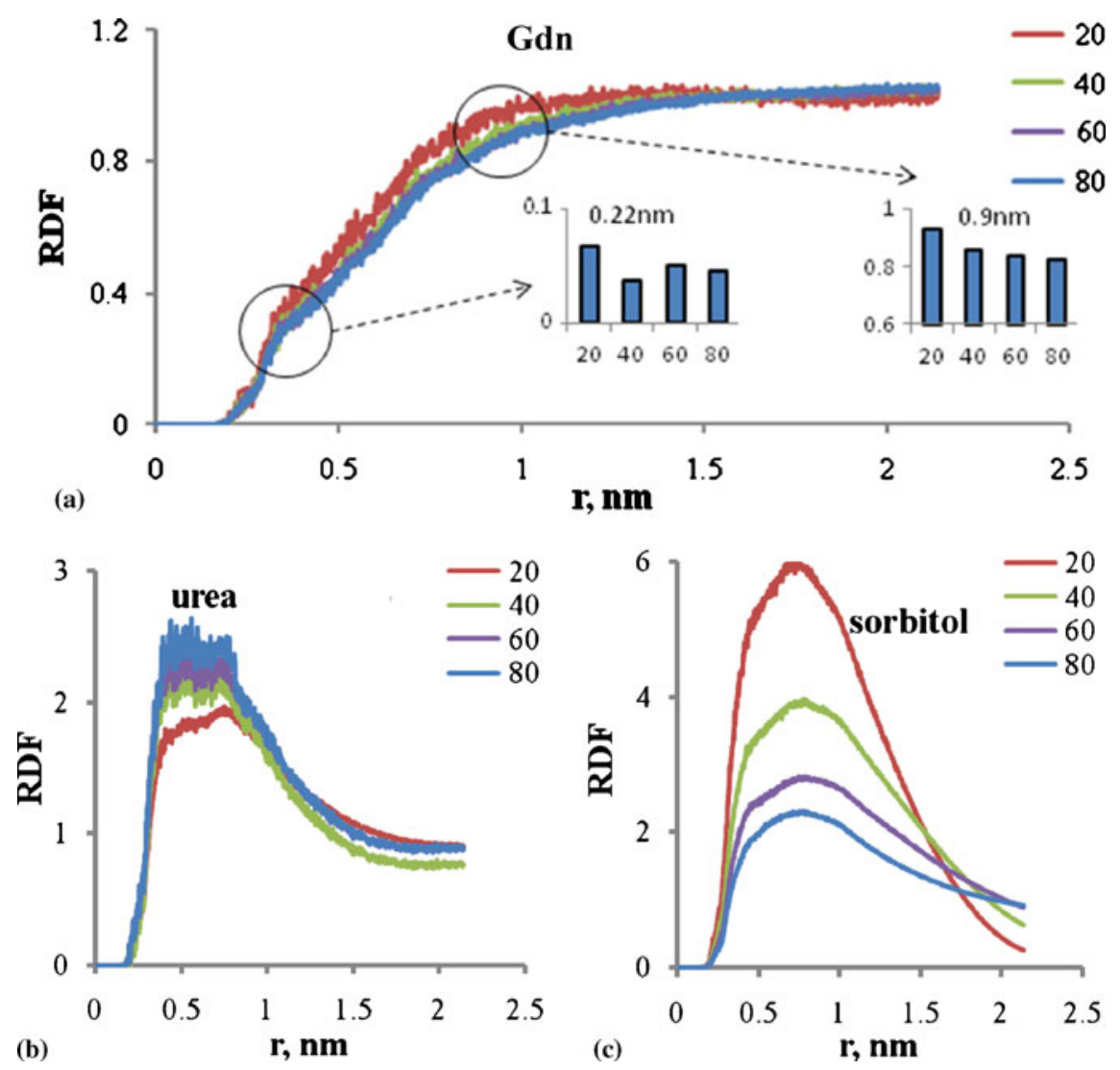

Figure 8. The radial distribution function of 20 to 80 . (a) Gdn, (b) urea and (c) sorbitol around porphyrin. 
Gdn and urea presence around porphyrin increase by concentration.

The radial distribution function of 20 to $80 \mathrm{Gdn}$, urea and sorbitol around porphyrin vs. distance were obtained and the results are shown in figure $8 \mathrm{a}-\mathrm{c}$, respectively. In order to explain this phenomenon, we have used the exclusion and preferential hydration concepts. This is the reason for stabilizing effect of osmolite compounds. Sorbitol can increase the hydration layer around porphyrin and Gdn decrease this layer. This effect is due to exclusion of sorbitol from solvent/porphyrin surface. In this work, preferential hydration has been investigated by analysing the RDF of Gdn, urea and sorbitol-solvent. It can be seen that by increasing Gdn concentration in $0.22 \mathrm{~nm}$, porphyrin preferentially becomes more hydrated. In long distances of $0.9 \mathrm{~nm}$ reduction of solvent molecules due to the presence of more Gdn molecules is resulted. So it can be concluded that a thin hydration layer on porphyrin surface exists in systems with low concentration of Gdn and urea (figure $8 \mathrm{a}, \mathrm{b}$ ). Figure $8 \mathrm{c}$ shows more reduction of RDF porphyrin-sorbitol. It means that sorbitol increases hydration layer around porphyrin in high concentrations without direct contact. This result is in good accordance with experimental results that in aqueous solutions, osmolites are excluded from the first hydration layer around porphyrin. ${ }^{27}$

The radial distribution function of chloride ion around porphyrin vs. distance is shown in figure 9 in various concentrations of Gdn. It can be seen that RDF

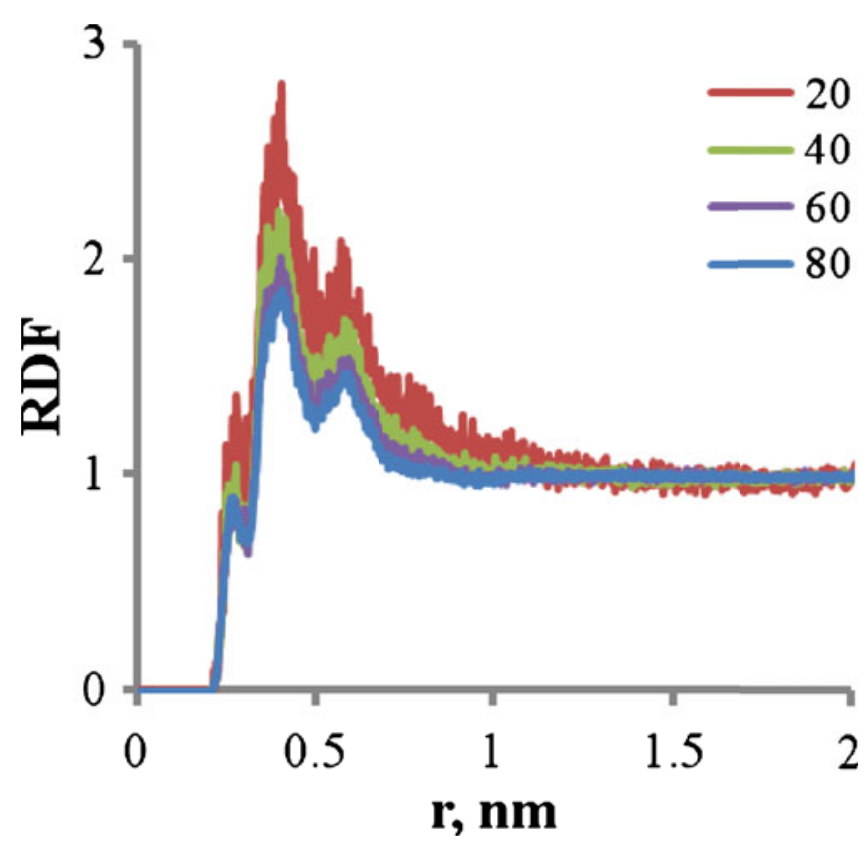

Figure 9. The radial distribution function of chloride ionGdn in various concentration of Gdn. is increased for Gdn in higher distances. This figure demonstrates that in distance $1.1 \mathrm{~nm}$ by increasing Gdn concentration, RDF of chloride ion around porphyrin increase but in $0.3 \mathrm{~nm}$, RDF decreases. It means that chloride ions tend to move toward porphyrins.

The radial distribution function of solvent around Gdn, urea and sorbitol vs. distance is shown in figure $10 \mathrm{a}-\mathrm{c}$ in various concentrations of $\mathrm{Gdn}$, urea and sorbitol, respectively. It can be seen that probability existence of solvent molecule near Gdn and urea is increased. It means that by increasing Gdn and urea concentration, probability existence of solvent molecule around Gdn and urea increases and RDF of sorbitol-solvent decrease by concentration.

RDF of Gdn-solvent in the 20 ns time interval in the presence of $20 \mathrm{Gdn}$, urea and sorbitol has been compared in figure 10d. It shows that probability existence of solvent molecule is more around Gdn, urea and sorbitol, respectively.

RDF of porphyrin-porphyrin has been obtained by molecular dynamics in the presence of 20 to $80 \mathrm{Gdn}$, urea and sorbitol and comparison of them has been done. Figure $11 \mathrm{a}-\mathrm{c}$ show the calculated RDF of porphyrin-porphyrin by molecular dynamics in the presence of 20 to $80 \mathrm{Gdn}$, urea and sorbitol. Results show more tendency of porphyrin to aggregate in the presence of sorbitol, urea and Gdn, respectively and by increasing Gdn and urea concentration, porphyrin aggregation decreases while porphyrin aggregation increases by increasing sorbitol content.

Occurrence probability of porphyrin around each other is compared and this probability is more in the presence of sorbitol, urea and Gdn. As a consequence, preferential hydration caused by sorbitol stabilizes the porphyrin aggregation.

The radial distribution function of chloride ionporphyrin in various concentration of Gdn has been obtained and result is shown in figure 12. This figure shows increase of RDF ion-porphyrin by increasing Gdn concentration. It demonstrates again that chloride ion move to porphyrin molecules.

Variation of distances between the mass centres of porphyrins versus time were obtained and then averaged from output of molecular dynamics simulation in the presence of Gdn, urea and sorbitol. Averaged distance was observed to increase with increasing concentration of urea and Gdn due to reduction of porphyrin aggregation and is depicted in figure 13. Table 1 gives the aggregation rate correspond to each concentration of porphyrin, so it can be seen that decrease in the aggregation of porphyrin was observed by increasing the number or concentration of Gdn and urea, indicating that they reduce aggregates formation and a reverse trend is 


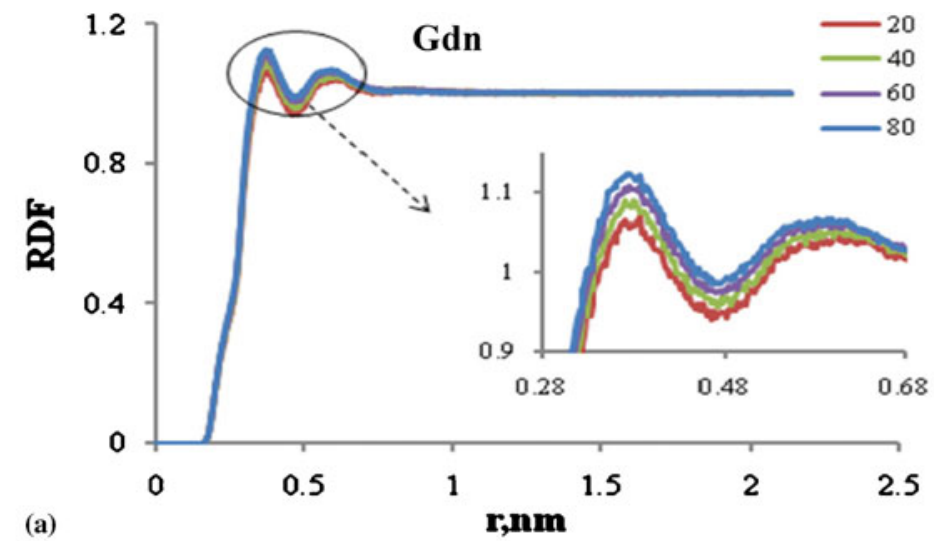

(a)
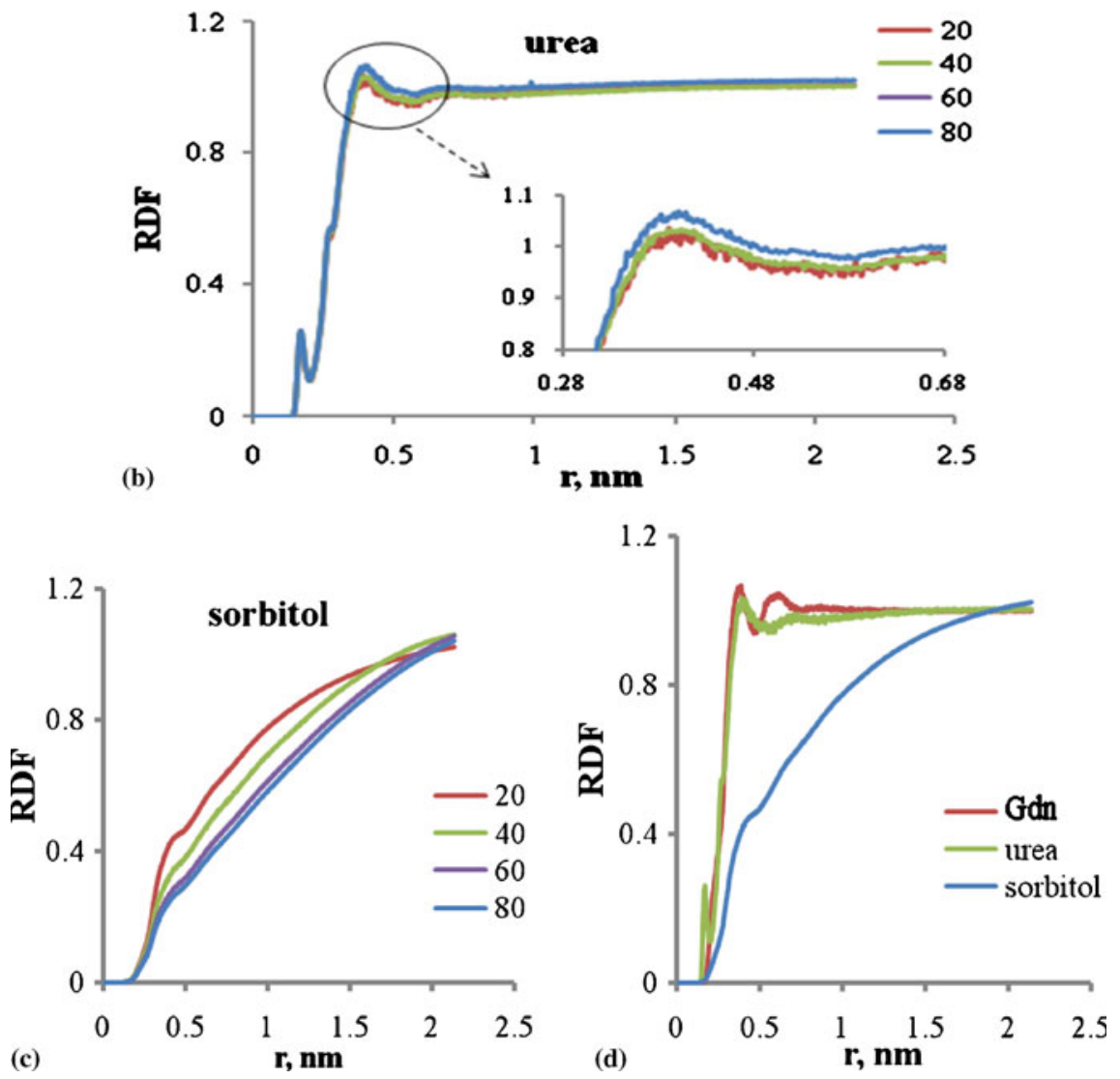

Figure 10. RDF of solvent around 20 to 80. (a) Gdn, (b) urea and (c) sorbitol in the $20 \mathrm{~ns}$ time interval. (d) Comparing RDF of solvent around $20 \mathrm{Gdn}$, urea and sorbitol.

observed for sorbitol. The aggregation rate varies with respect to concentration of solvent which is apparent in randomly selected diagrams.

Relaxation times and rate constants of porphyrin derivatives in each concentration of Gdn were calculated by transfer of distance data to Origin software. A detailed description of the $20 \mathrm{~ns}$ simulation includes the number of molecules, relaxation times and rate constant are presented in table 1. It is expected from above results that porphyrin forms aggregates in lower concentration of Gdn and more aggregation rate is observed in the presence of sorbitol. Comparison of Gdn and urea show more effect of Gdn to reduce porphyrin aggregation which confirms all above results. Results showed that, by increasing number from 20 to $80 \mathrm{M}$, rate constants decreases. It means that we have a decrease in rate constant and average porphyrin-porphyrin interaction. 

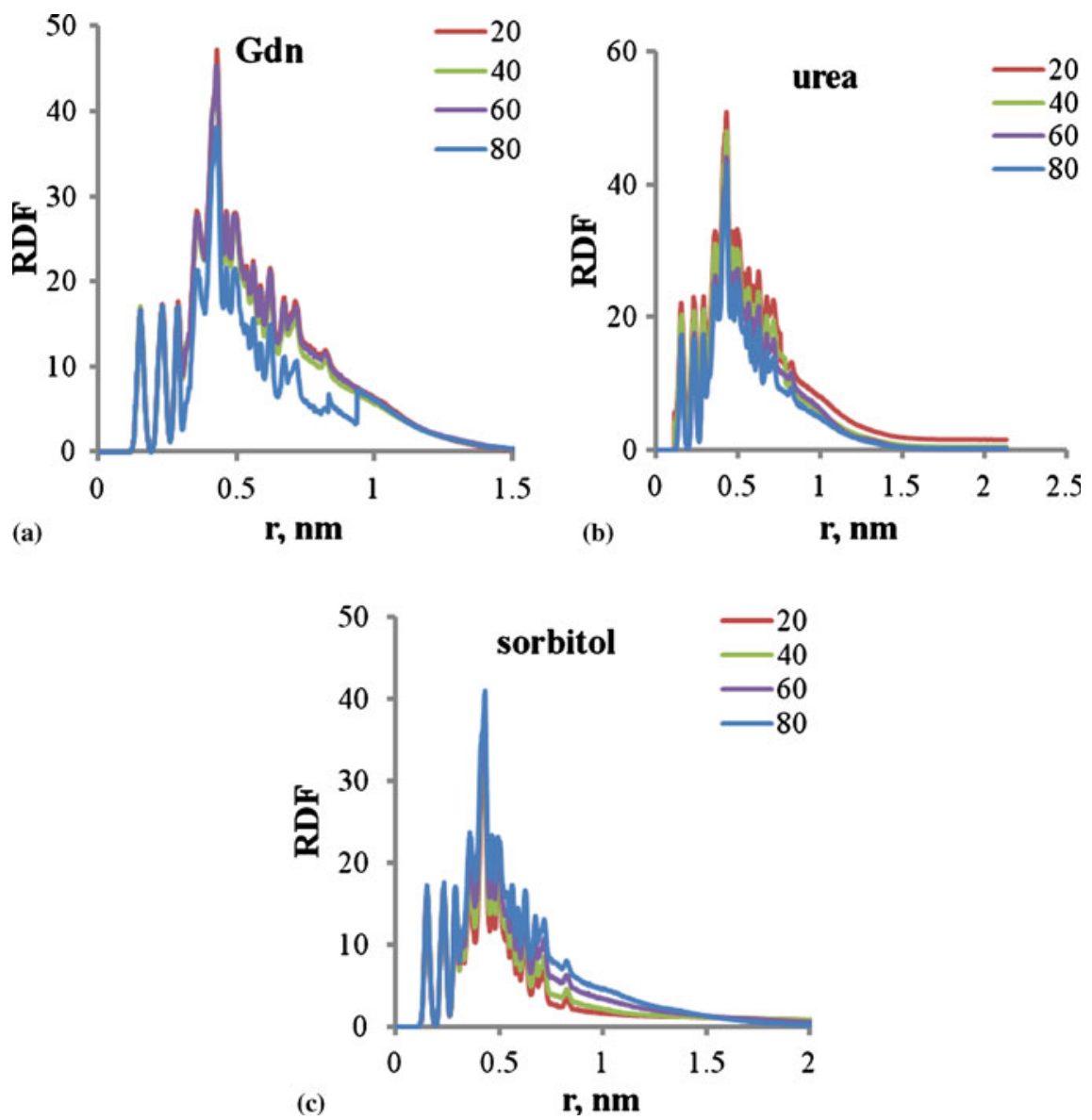

Figure 11. Calculated RDF of porphyrin-porphyrin by molecular dynamics in the presence of 20 to 80 molecules of (a) Gdn, (b) urea and (c) sorbitol.

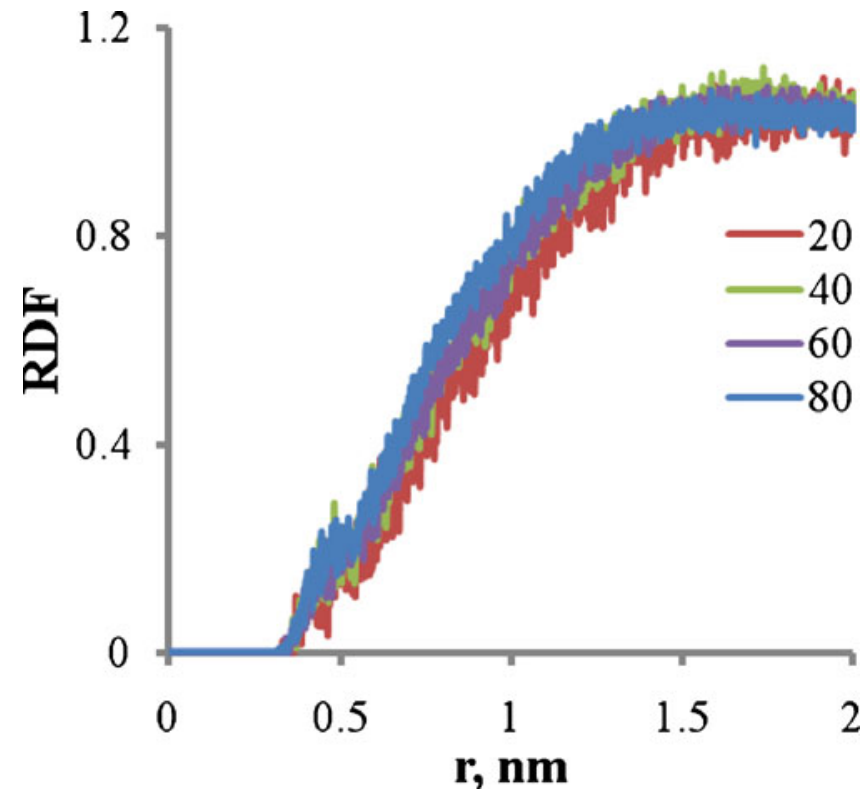

Figure 12. Calculated RDF of porphyrin-chloride ion by molecular dynamics in the presence of 20 to $80 \mathrm{Gdn}$.
Snapshots picture were taken by DS Visualizer program from the systems during $20 \mathrm{~ns}$ for 6 porphyrins in the presence of $20 \mathrm{Gdn}$ which are presented in figure 14 . Water molecules were omitted from the figure for clarity but Gdn molecules are also presented. By comparing the snapshots it is apparent that during $20 \mathrm{~ns}$, the aggregated form of porphyrin are dissolved in the solution in the presence of Gdn.

Porphyrin system in the presence of 20 molecules of Gdn, urea and sorbitol was simulated for $40 \mathrm{~ns}$ for comparison purpose and results of RMSD curves are presented in figure 15 . Results showed that 20 ns system equilibration was enough for our simulation because the systems do not have any special changes during final $30 \mathrm{~ns}$.

The figure also shows that system has more structural changes (RMSD) in the presence of Gdn than urea which confirms above results.

Binding of Gdn to porphyrin probably through hydrogen bonds between the Gdn group and porphyrin bonds was proposed. The affinity of guanidium being 

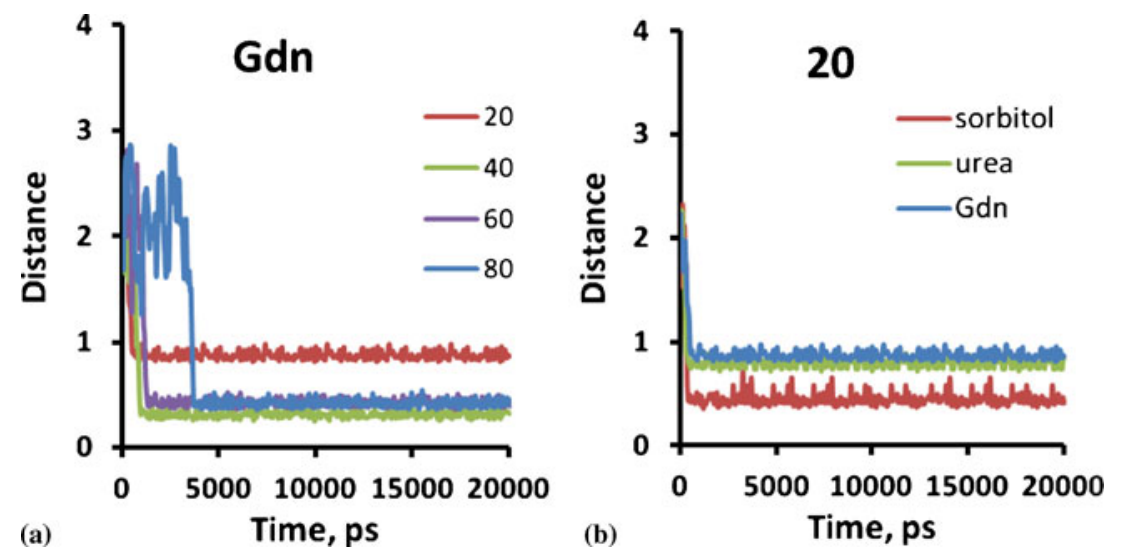

Figure 13. Variation of distance between porphyrins versus time for (a) 20 to $80 \mathrm{Gdn}$ and (b) $20 \mathrm{Gdn}$, urea and sorbitol.

Table 1. Results of relaxation time $(\tau)$ and rate constant (k) of porphyrin aggregation obtained from distance diagrams by Origin software.

\begin{tabular}{lcc}
\hline Num & $\tau$ & $\mathrm{k}$ \\
\hline $20 \mathrm{Gdn}$ & 0.003341 & 299.2841 \\
$40 \mathrm{Gdn}$ & 0.001664 & 600.9196 \\
$60 \mathrm{Gdn}$ & 0.001146 & 872.549 \\
$80 \mathrm{Gdn}$ & 0.000328 & 3052.481 \\
20 urea & 0.003609 & 277.1121 \\
20 sorbitol & 0.005150 & 194.180 \\
\hline
\end{tabular}

reduced by low concentration of guanidine which can stabilize the porphyrin in low concentrations. The binding of organic solvent to porphyrin through hydrophobic interaction explains the observed concentration dependence of the interaction. Here, Gdn acts as the strongest destabilizer of porphyrin aggregation. ${ }^{28-32}$

The mechanism for reduction and destabilization of porphyrin aggregation by Gdn and urea is explored by molecular dynamics simulation. Accumulation of urea around porphyrin shows that water molecules are expelled from the first hydration shell of the porphyrin.
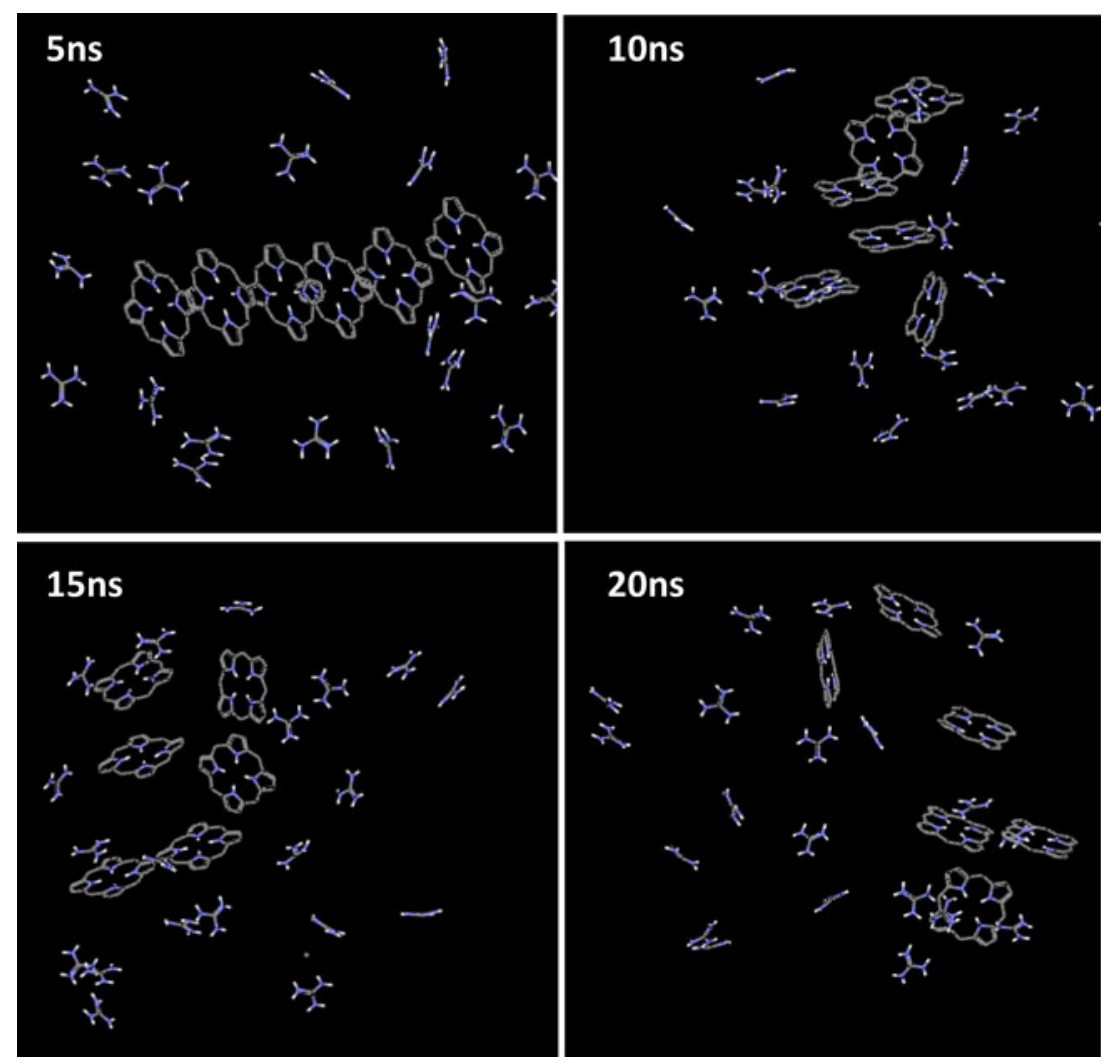

Figure 14. Snapshots picture was taken by DS Visualizer program from the 5 to $20 \mathrm{~ns}$ configurations of the porphyrin in the presence of $20 \mathrm{Gdn}$. 


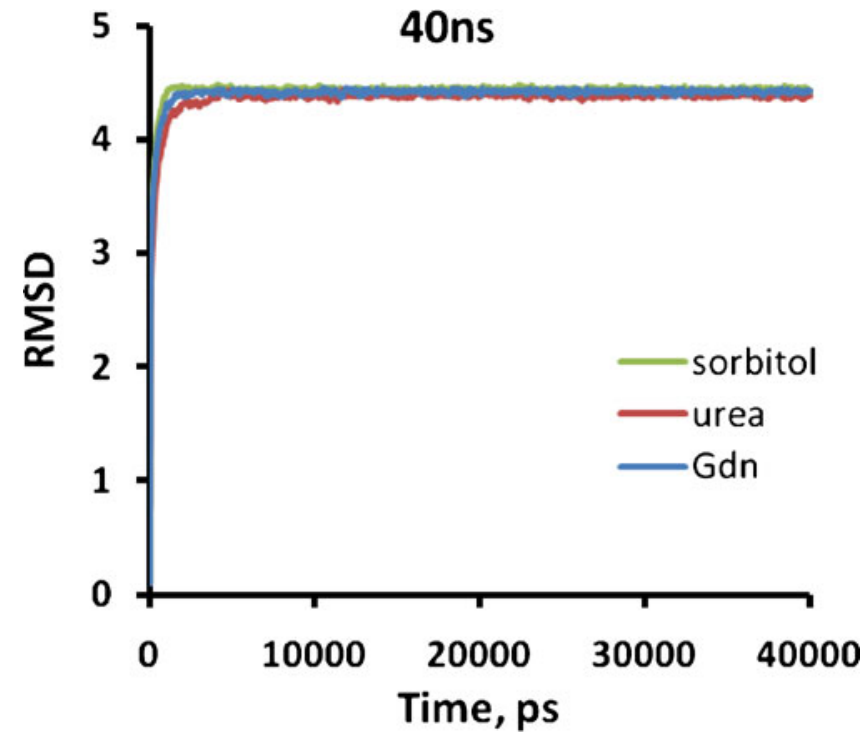

Figure 15. RMSD plot for porphyrin in the presence of 20 molecules of Gdn, urea and sorbitol was simulated for $40 \mathrm{~ns}$.

The direct dispersion interaction between urea and the porphyrin is stronger than indirect interaction, which gives rise to the intrusion of urea into the porphyrin. This is augmented by preferential hydrogen bond formation between the urea carbonyl and the nitrogen atom that contributes to porphyrin. Our study demonstrates that Gdn and urea has a stronger dispersion interaction with water than porphyrin.

Urea and Gdn molecules are presumed to bind to porphyrin and more porphyrin groups are exposed to these molecules. The differences in the urea and Gdn effect have been extensively discussed in terms of their differential binding to the porphyrin. Surprisingly, very little consideration has been attributed to the role of the differences in the ionic character between urea and Gdn in decrease of porphyrin aggregation. Twenty years ago, Greene and Pace reasoned that Gdn was 2.8 times more effective than urea. ${ }^{18}$

At low concentration, Gdn act as stabilizer due to its ionic nature. In comparison with osmolites, urea and Gdn decrease the hydration layer of porphyrin and sorbitol as stabilizer factor increase this layer. In high concentrations Gdn has less hydration tendency and move towards porphyrin and interact with it while this is not true for osmolites and in high concentrations they tend to aggregate and also increase the hydrated layer.

\section{Conclusions}

Small organic molecules such as Gdn, urea and sorbitol in aqueous solution can have profound effects on porphyrin aggregation and function. The use of these solutions to stabilize or destabilize porphyrin aggregation, depending on the co-solvent is common. Destabilization, with an agent such as urea or Gdn, is one of the primary ways to assess porphyrin aggregation stability. Despite its widespread use, the molecular basis for Gdn and urea's ability to destabilize aggregation remains unknown. We demonstrated here that Gdn and urea exert their effect directly, by binding to the porphyrin and reduction of hydration layer around porpyrin and sorbitol effect indirectly, by altering the solvent environment. Most versions of the direct interaction model assume that urea binds to model molecule, and stabilizes the monomer state, thereby favouring monomer formation. Similar but stronger destabilization effect is seen for Gdn. Unfortunately, it seems unlikely that experimental approaches will provide the molecular details of how urea destabilizes porphyrin aggregation, so we have employed molecular dynamics (MD) simulations to address this issue.

\section{Acknowledgements}

The financial support of Damghan University is acknowledged.

\section{References}

1. Bonnett R 1995 Chem. Soc. Rev. 2419

2. Collings P J, Gibbs E J, Starr T E, Vafek O, Yee C, Pomerance L A and Pasternack R F 1999 J. Phys. Chem. B 1038474

3. Jori G and Spikes J D 1984 Photobiochemistry of porphyrins, K C Smith (ed.), Topics in photomedicine, New York: Plenum, 183

4. Dougherty T J and Marcus S L 1992 Eur. J. Cancer 28 1734

5. Moan J and Berg K 1992 Photochem. Photobiol. 55931

6. Grand A and Gasparro F 1993 Spectrum 628

7. Gandini S C M, Borissevitch I E, Perussi J R, Imasato H and Tabak M 1998 Luminescence 7853

8. Kano K, Minamizono H, Kitae T and Negi S 1997 J. Phys. Chem. A 1016118

9. Kano K, Fukuda K, Wakami H, Nishiyabu R and Pasternack R F 2000 J. Am. Chem. Soc. 1227494

10. Rahman M and Harmon H 2007 J. Phys. A 11125

11. Ghadamgahi M and Ajloo D 2011 J. Porphyr. Phthalocya. 15240

12. Perepogu A K and Bangal P R 2008 J. Chem. Sci. 120 485

13. Pasternack R F, Fleming C, Herring S, Collings $\mathrm{P}$ J, dePaula J, DeCastro G and Esther J 2000 Gibbs. Biophys. J. 79550

14. Alonso D O V and Daggett V 1995 J. Mol. Biol. 247501

15. Caflisch A and Karplus M 1999 Struct. Fold. Des. 7477

16. Laidig K E and Daggett V 1996 J. Phys. Chem. 1005616 
17. Agarwal M and Chakravarty C 2009 J. Chem. Sci. 121 913

18. Wetlaufer D, Malik S, Stoller L and Coffin R 1964 J. Am. Chem. Soc. 86508

19. Frank H and Franks F 1968 J. Chem. Phys. 484746

20. Hammes G G and Schimmel P R 1967 J. Am. Chem. Soc. 89442

21. Dwek R, Luz Z and Shores M 1970 J. Phys. Chem. 74 2230

22. Bennion B and Daggett V 2003 Proc. Natl. Acad. Sci. USA 1005142

23. Auton M, Holthauzen L M F and Bolen D W 2007 Proc. Natl. Acad. Sci. USA 12231

24. OBrien E, Dima R, Brooks B and Thirumalai D 2007 J. Am. Chem. Soc. 1297346
25. http://manual.gromacs.org/

26. Mason P E, Neilson G W, Dempsey C E, Barnes A C and Cruickshank J M 2003 Proc. Natl. Acad. Sci. USA 1004557

27. Liu F F, Ji L, Dong X Y and Sun Y 2009 J. Phys. Chem. B 11311320

28. deOliveira O V, deMoura A F and Freitas L C G 2007 J. Mol. Struct: Theochem. $\mathbf{8 0 8} 93$

29. Arakawa T R, Bhat R S N and Timasheff S N 1990 J. Biochem. 291914

30. Kita Y, Arakawa T, Lin T Y and Timasheff S N 1994 J. Biochem. 3315178

31. Fitter J and Pohlmeier S H 2004 J. Biochem. 439589

32. Huaa L, Zhoua R, Thirumalaic D and Bernea B J 2008 Proc. Natl. Acad. Sci. USA 10516928 